\title{
Thioredoxin-interacting protein is an independent risk stratifier for breast ductal carcinoma in situ
}

\author{
Islam M. Miligy $\mathbb{1}^{1,2} \cdot$ Kylie L. Gorringe $\mathbb{1}^{3,4} \cdot$ Michael S. Toss ${ }^{1,5} \cdot$ Abdulbaqi A. Al-Kawaz ${ }^{1}$ Peter Simpson $\mathbb{D}^{6} \cdot$ \\ Maria Diez-Rodriguez ${ }^{1}$ - Christopher C. Nolan ${ }^{1} \cdot \operatorname{lan}$ O. Ellis ${ }^{1} \cdot$ Andrew R. Green ${ }^{1} \cdot$ Emad A. Rakha ${ }^{1,2}$
}

Received: 12 January 2018 / Revised: 21 May 2018 / Accepted: 23 May 2018 / Published online: 28 June 2018

(c) United States \& Canadian Academy of Pathology 2018

\begin{abstract}
Current clinicopathological parameters are useful predictors of breast ductal carcinoma in situ behavior, but they are insufficient to define high-risk patients for disease progression precisely. Thioredoxin-interacting protein (TXNIP) is a key player of oxidative stress. This study aims to evaluate the role of TXNIP as a predictor of ductal carcinoma in situ progression. Tissue microarrays from 776 pure ductal carcinoma in situ and 239 mixed ductal carcinoma in situ and invasive tumors were constructed. All patients were treated at a single institution with a long-term follow-up and TXNIP expression was assessed using immunohistochemistry. TXNIP expression was investigated in terms of associations with clinicopathological and molecular features and patient outcome. Loss/reduced cytoplasmic expression of TXNIP was associated with features of aggressiveness including high nuclear grade $\left(p=1.6 \times 10^{-5}\right)$, presence of comedo necrosis $(p=$ $0.001)$, and estrogen receptor negative (ER-)/HER2 - ductal carcinoma in situ $\left(p=4.6 \times 10^{-5}\right)$. Univariate analysis showed an inverse association between TXNIP expression and outcome in terms of shorter local recurrence-free survival $(p=$ 0.009). Multivariable analyses showed that independent predictors of ductal carcinoma in situ recurrence were low TXNIP expression $(p=0.005, \mathrm{HR}=0.51$, and 95\% CI: 0.32-0.81), larger ductal carcinoma in situ size, and high nuclear grade. TXNIP functions as a tumor suppressor gene with loss of its expression associated with ductal carcinoma in situ recurrence. TXNIP can be used as a potentially useful marker in prognostic stratification of ductal carcinoma in situ for management decisions.
\end{abstract}

Electronic supplementary material The online version of this article (https://doi.org/10.1038/s41379-018-0086-7) contains supplementary material, which is available to authorized users.

Emad A. Rakha

emad.rakha@nottingham.ac.uk

Emad.rakha@nuh.nhs.uk

1 Academic Pathology, Division of Cancer and Stem Cells, School of Medicine, The University of Nottingham, Nottingham City Hospital, Nottingham, UK

2 Histopathology Department, Faculty of Medicine, Menoufia University, Shibin El Kom, Egypt

3 Cancer Genomics Program, Peter MacCallum Cancer Centre, Melbourne, VIC, Australia

4 The Sir Peter MacCallum Department of Oncology, University of Melbourne, Parkville, VIC, Australia

5 Histopathology Department, South Egypt Cancer Institute, Assiut University, Assiut, Egypt

6 Centre for Clinical Research, Faculty of Medicine, The University of Queensland, Brisbane, QLD, Australia

\section{Introduction}

The main aim of ductal carcinoma in situ management is to prevent local recurrence particularly invasive recurrence [1]. Despite a low mortality risk, ductal carcinoma in situ is often treated with mastectomy or breast conservative surgery with frequent re-excisions compared to invasive disease [2]. Mastectomy results in very few recurrences but is often considered as over-treatment for screen-detected precursor lesions. Conversely, breast conservative surgery alone increases the risk of recurrence, which may be considered as under-treatment in a proportion of high-risk ductal carcinoma in situ [3]. Radiotherapy can reduce the recurrence rate by approximately $50 \%$ but has significant side effects and its application should therefore be rationalized. Accurate risk stratification of ductal carcinoma in situ means not only predicting recurrence but also which patients are likely to progress to invasive disease.

Ductal carcinoma in situ is observed in more than half of invasive breast cancer [4] and in the majority of these cases, 
they share morphology, immunoprofile, and genetic features suggesting that the invasive component arises from the associated ductal carcinoma in situ [5-7]. However, there remains a lack of objective predictive markers of ductal carcinoma in situ behavior; not only of recurrence but also development of invasive disease that could aid in treatment decisions.

Clinicopathological characteristics are used as prognostic factors in guiding treatment decisions [3] but they are insufficient to reflect the molecular and clinical heterogeneity. Assessment of molecular markers can be used as surrogates of ductal carcinoma in situ biology and behavior and have the potential to predict patient outcome [8]. It is known that cancer cells continuously experience oxidative stress, resulting from heightened reactive oxygen species generation [9]. Enhanced oxidative stress has been implicated in the initiation and progression of cancer, promoting cell survival and drug resistance in some cases [10]. However, unresolved high levels of oxidative stress beyond the capacity of the cancer cell to manage causes critical damage to cellular DNA, proteins, and lipids ultimately leading to cell death [11]. A negative regulator of the major antioxidant thioredoxin (TRX), TRX-interacting protein (TXNIP), has potent growth suppressive, metastasis inhibitory and proapoptotic functions [12]. Loss of TXNIP expression was reported to be associated with the development of some solid tumors [12]. In the breast, high expression of TXNIP was reported in normal breast tissues [13] and showed reduced immunointensity in invasive tumors [14]. Ectopic expression of TXNIP in the breast cancer cell line MCF-7 drives cells to undergo senescence, accompanied by increased reactive oxygen species release. In a study of 98 locally advanced invasive breast cancer treated with anthracycline-based chemotherapy, Woolston et al. [15] have demonstrated that TXNIP is an independent predictor of outcome.

We hypothesized that oxidative stress could play a role in breast cancer progression and that TXNIP might be a potential prognostic marker in ductal carcinoma in situ. In this study, TXNIP expression was assessed in a large $(n=1015)$ and well-characterized cohort of ductal carcinoma in situ from a single institution with long-term follow-up. In addition, the prognostic significance of TXNIP gene copy number and gene expression was assessed in a large cohort of invasive breast cancer $(n=1980)$ as a molecular surrogate of ductal carcinoma in situ.

\section{Patients and methods}

\section{Study cohort}

This retrospective study was conducted on a consecutive series of 1059 primary pure ductal carcinoma in situ cases diagnosed, and treated in a uniform manner, between
1990 and 2012 at the Nottingham City Hospital, Nottingham, UK. Exclusion of referral, miscoded, and recurrent cases resulted in 776 pure primary ductal carcinoma in situ with available formalin-fixed paraffinembedded tumor blocks for tissue microarray construction. A series of 239 cases diagnosed as synchronous ductal carcinoma in situ and invasive tumors (mixed ductal carcinoma in situ and invasive breast cancer) was also collected as a validation set. Patients' demographic information, histopathological parameters, management, radiotherapy, and patient outcome data were collated. No patients were treated with hormonal therapy. Local recurrence-free survival was calculated based on the time (in months) from the date of primary surgical treatment to the time of ipsilateral local recurrence. The median follow-up period, in pure ductal carcinoma in situ cohort, was 118 months (range 2-240 months), during which 90 patients (14\%) developed local ipsilateral recurrence, including invasive $(60 / 90 ; 67 \%)$ or ductal carcinoma in situ $(30 / 90 ; 33 \%)$. Recurrence following breastconserving surgery and mastectomy occurred in 84 and 6 cases, respectively.

Patients who developed contralateral disease following ductal carcinoma in situ diagnosis were censored at the time of diagnosis of the contralateral cancer.

\section{Tissue microarray and immunohistochemistry}

Tissue microarrays were prepared from representative ductal carcinoma in situ lesions of the pure cases and from ductal carcinoma in situ and invasive tumors from the mixed cases. The tissue microarray was constructed using three-dimensional Histech ${ }^{\circledR}$ Grand Master ${ }^{\circledR}$, whereby cores of 1 and $0.6 \mathrm{~mm}$ were taken from ductal carcinoma in situ and invasive tumor samples, respectively. In addition, a set of whole tissue sections from 10 cases containing ductal carcinoma in situ and invasive tumors were assessed to evaluate heterogeneity and the pattern of TXNIP expression in normal and malignant breast lesions.

Validation of TXNIP antibody (Abcam; clone EPR14774) specificity was performed using western blotting on whole-cell lysates of MCF-7, SKBr3, and MDAMB-231 human breast cancer cell lines (obtained from the American Type Culture Collection; Rockville, MD, USA). TXNIP antibody was used at 1:3000 dilution, which showed a single specific band at the predicted size of $44 \mathrm{kDa}$ (Fig. 1a).

Expression of TXNIP protein in ductal carcinoma in situ was assessed by immunohistochemistry using the Novocastra Novolink polymer detection system (Leica, Newcastle, UK). Four-micrometer tissue microarray and full-face sections were stained with mouse monoclonal TXNIP antibody (1:3000) for 30 min. 3,30-Diaminobenzidine tetrahydrochloride (Novolink 
A
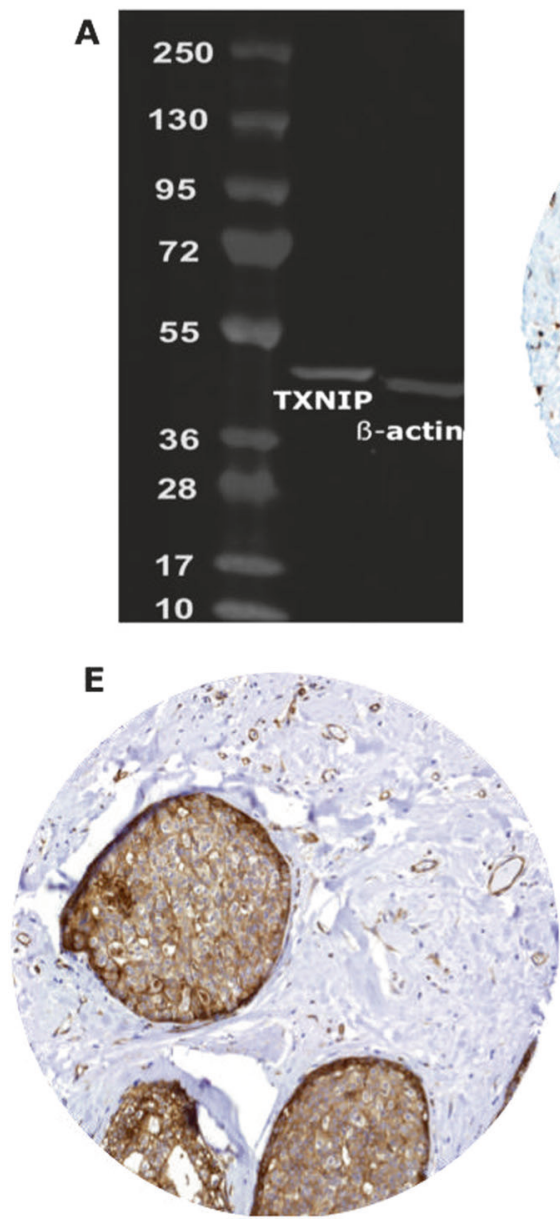

Fig. 1 Characterization of TXNIP expression in ductal carcinoma in situ. a Western blot analysis of the anti-TXNIP antibody confirmed a single specific band at $44 \mathrm{kDa}$ in a variety of cell lines, image for MCF-7 shown, b TXNIP cytoplasmic expression in normal breast tissue showing expression in the epithelium and a few scattered myoepithelial cells. Occasional membrane and/or nuclear staining is also seen. TXNIP immunohistochemistry expression in ductal

DAB substrate buffer) was used as a chromogenic substance. Sections were counterstained with hematoxylin. Positive staining controls (human tonsil and kidney tissue) were included while a negative control was achieved by omitting the application of the primary antibody. Expression of estrogen receptor (ER) in ductal carcinoma in situ was carried out using ER clone SP1, Ventana Benchmark ${ }^{\oplus}$ ULTRA system (Tucson, Arizona, USA), as per the recommended protocol (Supplementary material 1). A cutoff of $\geq 1 \%$ for ER staining positivity was used [16]. HER2 status was assessed using immunohistochemistry staining (1:400, DAKO, no antigen retrieval) with HercepTest scoring method as previously published $[16,17]$ (Supplementary material 1). In equivocal cases (HER2 immunohistochemistry score 2+), HER2 gene amplification was determined by chromogenic in situ hybridization using ZytoDot 2CSPEC ERBB2/CEN 17 Probe Kit (Supplementary material 2) [17, 18].
C

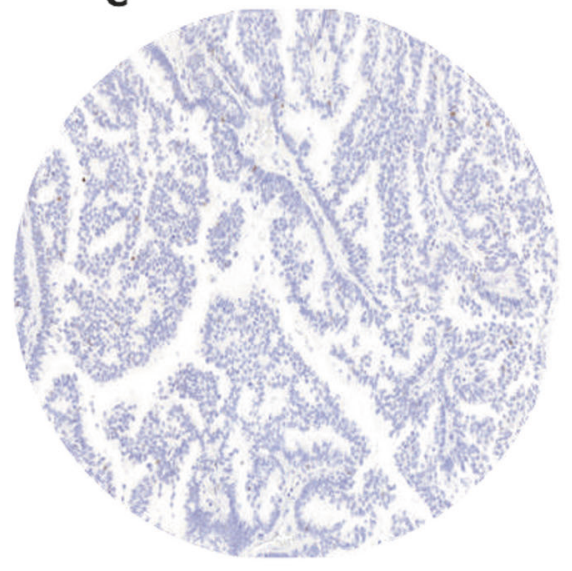

G

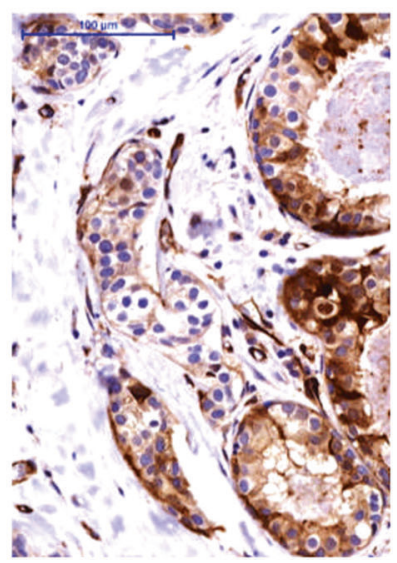

carcinoma in situ showing variable intensities from negative (c), weak (d), moderate (e), and strong (f) staining ( $\times 20$ magnification). g Fullface section of a ductal carcinoma in situ case mixed with invasive carcinoma and show moderate TXNIP expression in ductal carcinoma in situ but reduced/negative expression in the adjacent invasive carcinoma component

Cytoplasmic TXNIP staining was assessed using the semiquantitative $H$-score taking into consideration the intensity of staining and the percentage of stained tumor cells within each tissue core [19]. All cases were scored blinded to clinicopathological and outcome data. Cases with multiple cores $(n=210)$ were scored and the average was used as the final score.

This work obtained ethics approval by the North WestGreater Manchester Central Research Ethics Committee under the title; Nottingham Health Science Biobank, reference number $15 / \mathrm{NW} / 0685$.

\section{Analysis of TXNIP in invasive breast cancer}

To confirm the prognostic and clinical significance of TXNIP in invasive breast cancer, as a molecular surrogate of ductal carcinoma in situ in the breast, TXNIP gene copy 
Table 1 Correlation between TXNIP expression and the clinicopathologic variables of pure ductal carcinoma in situ cases

\begin{tabular}{llll}
\hline Parameter & \multicolumn{3}{l}{ TXNIP expression } \\
\cline { 2 - 4 } & $n$ High & Low & $\chi^{2}$ \\
& $(n=225)$ & $(n=411)$ & $(p$ value $)$ \\
& $n(\%)$ & $n(\%)$ & \\
\hline
\end{tabular}

\begin{tabular}{|c|c|c|c|c|}
\hline \multicolumn{5}{|l|}{ Age, years ${ }^{\mathrm{a}}$} \\
\hline$<40$ & $23(3.6)$ & $10(5)$ & $13(4)$ & 0.71 \\
\hline Between 40 and 60 & $\begin{array}{l}371 \\
(58.3)\end{array}$ & $131(58)$ & $240(58)$ & $(0.702)$ \\
\hline More than 60 & $\begin{array}{l}242 \\
(38.1)\end{array}$ & $84(37)$ & $158(38)$ & \\
\hline
\end{tabular}

Presentation

$\begin{array}{lllll}\text { Screening } & 302 & 113(50) & 221(54) & 0.73 \\ & (47.5) & & & \\ \text { Symptomatic } & 334 & 112(50) & 190(46) & (0.391) \\ & (52.5) & & & \end{array}$

Ductal carcinoma in situ size $(\mathrm{mm})$

$\begin{array}{lllll}<16 & \begin{array}{l}218 \\ (34.3)\end{array} & 84(37) & 134(33) & 4.73 \\ \text { Between } 16 \text { and } 40 & \begin{array}{l}246 \\ (38.7)\end{array} & 80(36) & 166(40) & (0.098) \\ & & & \\ \text { More than 40 } & \begin{array}{l}167 \\ (26.3)\end{array} & 57(25) & 110(27) & \end{array}$

Nuclear grade

$\begin{array}{lllll}\text { Low } & 87(13.7) & 50(22) & 37(9) & 24.88 \\ \text { Intermediate } & 162 & 61(27) & 101(25) & (\mathbf{1 . 6} \times \\ & (25.5) & & & \left.\mathbf{1 0}^{-5}\right) \\ \text { High } & 387 & 114(51) & 273(66) & \\ & (62.8) & & & \end{array}$

Ductal carcinoma in situ histologic type

$\begin{array}{lllll}\text { Single } & 312 & 113(50) & 199(48) & 0.189 \\ & (49.1) & & & \\ \text { Mixture }^{\mathrm{b}} & 324 & 112(50) & 212(52) & (0.664) \\ & (50.9) & & & \end{array}$

Comedo necrosis

$\begin{array}{lllll}\text { Yes } & 403 & 122(54) & 281(68) & 12.45 \\ & (63.4) & & & \\ \text { No } & 233 & 103(46) & 130(32) & (\mathbf{0 . 0 0 1}) \\ & (36.6) & & & \end{array}$

Coexistent LCIS

$\begin{array}{lllll}\text { Yes } & 58(9.1) & 21(9) & 37(9) & 0.02 \\ \text { No } & 578 & 204(91) & 374(91) & (0.890) \\ & (90.9) & & & \end{array}$

Coexistent Paget's

$\begin{array}{lllll}\text { Yes } & 31(9) & 7(6) & 24(11) & 2.42 \\ \text { No } & 304(51) & 102(94) & 202(89) & (0.463) \\ \begin{array}{l}\text { Management } \\ \text { Mastectomy }\end{array} & \begin{array}{l}323 \\ (50.7)\end{array} & 110(49) & 213(52) & 3.56 \\ & & & \\ \begin{array}{l}\text { Breast-conserving } \\ \text { surgery }\end{array} & \begin{array}{l}313 \\ (49.3)\end{array} & 115(51) & 198(48) & (0.207) \\ & & & & \end{array}$

Table 1 (continued)

\begin{tabular}{|c|c|c|c|c|}
\hline \multirow[t]{2}{*}{ Parameter } & \multicolumn{4}{|c|}{ TXNIP expression } \\
\hline & $n(\%)$ & $\begin{array}{l}\text { High } \\
(n=225) \\
n(\%)\end{array}$ & $\begin{array}{l}\text { Low } \\
(n=411) \\
n(\%)\end{array}$ & $\begin{array}{l}\chi^{2} \\
(p \text { value })\end{array}$ \\
\hline \multicolumn{5}{|l|}{ Radiotherapy $^{\mathrm{c}}$} \\
\hline Yes & $96(15.1)$ & $35(16)$ & $61(15)$ & 0.06 \\
\hline No & $\begin{array}{l}540 \\
(84.9)\end{array}$ & $190(84)$ & $350(85)$ & $(0.516)$ \\
\hline \multicolumn{5}{|c|}{ Estrogen receptor status } \\
\hline Positive & $412(75)$ & $150(87)$ & $262(69)$ & 22.46 \\
\hline Negative & $137(25)$ & $22(13)$ & $115(31)$ & $\left(2 \times 10^{-6}\right)$ \\
\hline \multicolumn{5}{|l|}{ HER2 status ${ }^{\mathrm{d}}$} \\
\hline Negative & $471(80)$ & $178(85)$ & $293(76)$ & 12.15 \\
\hline Positive & $120(20)$ & $31(15)$ & $89(23)$ & $(0.007)$ \\
\hline \multicolumn{5}{|c|}{ Molecular classification } \\
\hline ER +/HER2- & $\begin{array}{l}342 \\
(53.8)\end{array}$ & $139(79)$ & $203(60)$ & 24.02 \\
\hline ER-/HER2+ & $61(9.6)$ & $12(7)$ & $49(14)$ & $\begin{array}{l}(4.6 \times \\
\left.10^{-5}\right)\end{array}$ \\
\hline $\mathrm{ER}+/ \mathrm{HER} 2+$ & $43(6.8)$ & $14(8)$ & $29(9)$ & \\
\hline ER -/HER2- & $66(10.4)$ & $10(6)$ & $56(17)$ & \\
\hline
\end{tabular}

$p$ value in bold: significant

TXNIP thioredoxin-interacting protein, $n$ number, LCIS lobular carcinoma in situ

${ }^{a}$ Age: categorized according to the Van Nuys Prognostic Index (VNPI)

${ }^{\mathrm{b}}$ Histologic type is a mixture of more than one morphologic type

${ }^{\mathrm{c}}$ In all, 93 cases who were treated with radiotherapy belong to breastconserving surgery group, the remaining 3 cases were treated with mastectomy

${ }^{\mathrm{d}}$ HER2 final status is achieved using combination of IHC and chromogenic in situ hybridization (CISH)

number and normalized gene expression (mRNA) data were analyzed using the Molecular Taxonomy of Breast Cancer International Consortium (METABRIC) dataset that comprises 1980 tumors with long-term follow-up [20].

\section{Statistical analysis}

Statistical analyses were performed using SPSS v23 (Chicago, IL, USA) for Windows. X-tile software program (Yale University, version 3.6.1) was utilized to define the optimal cutoff point for TXNIP expression $(H$-score 120$)$ with corrected $p$ value and relative risk against local recurrence-free survival [21, 22]. Association between TXNIP expression and clinicopathological parameters using categorized data was evaluated using Chi-squared test. Survival rates were determined using the Kaplan-Meier method and compared by the log-rank test. Multivariate analysis using Cox proportional hazard regression model determined the influence of TXNIP expression, when 
adjusted to other variables, on local recurrence-free survival. All tests were two-tailed and a $p$ value of $<0.05$ was considered as statistically significant.

\section{Results}

Analysis of TXNIP mRNA levels in invasive breast cancer revealed significant association between reduced levels/loss of expression and high histological grade $(p=0.004)$, large tumor size $(p=0.003)$, advanced-stage tumors $(p=0.01)$, and with ER-negative and HER2-positive phenotype ( $p=$ 0.003 and 0.011 , respectively) and luminal B and basal intrinsic subtypes according to the PAM50 classification $\left(p=6.7 \times 10^{-27}\right)($ Supplementary Table 1$)$. Loss of copy number and reduced TXNIP mRNA expression were associated with worse outcome in terms of shorter breast cancer-specific survival ( $p=0.003$ and 0.002 , respectively) (Supplementary Figure 1). Reduced TXNIP mRNA expression was also associated with loss of copy number $(p=0.049)$. These findings confirmed the tumor suppressor function of TXNP in breast cancer and its potential prognostic value and supported investigating its role in ductal carcinoma in situ.

\section{Frequency and localization pattern of TXNIP in ductal carcinoma in situ}

Assessment of whole tissue sections revealed cytoplasmic expression of TXNIP in a homogenous distribution pattern with occasional nuclear staining in normal luminal and myoepithelial cells. The homogenous pattern of expression confirmed the validity of using tissue microarray technology to assess its expression. Variable staining intensities and localization was detected in the different morphological components, with occasional strong nuclear staining in normal breast epithelial cells, strong to moderate cytoplasmic staining in ductal carcinoma in situ (with occasional membrane accentuation), and mild to negative cytoplasmic staining in invasive tumors (with no nuclear staining). TXNIP-positive pure ductal carcinoma in situ tumor cells exhibited distinct cytoplasmic staining with occasional peripheral/perimembrane accentuation and infrequent nuclear staining in some low-grade tumors (Fig. 1). After exclusion of uninformative cores (i.e., loss and/or folding of cores), a total of 636 pure ductal carcinoma in situ and 231 tumors with mixed ductal carcinoma in situ and invasive components were included in the analysis. TXNIP expression showed a unimodal distribution with a median $H$-score of 185 (range $0-300$ ) in the pure ductal carcinoma in situ.

In mixed ductal carcinoma in situ and matched invasive cancer, both components were consistently positive in 27
(12\%) cases and negative in $65(28 \%)$ cases. High ductal carcinoma in situ/low invasive breast cancer expression for TXNIP was present in 134 (58\%) cases however, there were $5(2 \%)$ cases in which invasive breast cancer was positive/ high expressing and ductal carcinoma in situ low expressing for TXNIP. When pure ductal carcinoma in situ was compared to ductal carcinoma in situ mixed with invasive breast cancer, a statistically significant difference was detected, with the TXNIP cytoplasmic mean $H$-score being higher in pure ductal carcinoma in situ tumors $(\bar{X} \pm \mathrm{SD}=185 \pm 76$ and $112 \pm 88$, respectively; $t$-test $=15.8$ and $p<0.0001$ ). In the mixed cases, a significant difference was also observed whereby the TXNIP cytoplasmic mean $H$-score was higher in the ductal carcinoma in situ component when compared with the adjacent invasive component $(\bar{X} \pm \mathrm{SD}=112 \pm 88$ and $83 \pm 78$, respectively; $Z=16.6$ and $p<0.0001$ ) (Supplementary Figure 2). Similar results were observed when TXNIP was analyzed as a categorical variable (Chi-squared $p<0.001$ for pure vs mixed ductal carcinoma in situ and $p<0.001$ for ductal carcinoma in situ vs adjacent invasive components). No nuclear expression was seen in tumor cells of the cases mixed with invasion.

\section{Association of TXNIP with clinicopathological parameters in pure ductal carcinoma in situ}

High/positive TXNIP cytoplasmic expression was significantly associated with good prognostic factors in ductal carcinoma in situ, including low nuclear grade $\left(p=1.6 \times 10^{-5}\right)$, absence of comedo-type necrosis $(p=$ $0.001)$, positive ER status $\left(p=2 \times 10^{-6}\right)$, negative HER2 status $(p=0.007)$, and with the luminal $\left(\right.$ ER+/HER2-) phenotype $\left(p=4.6 \times 10^{-5}\right)($ Table 1$)$.

\section{Association with patient outcome of pure ductal carcinoma in situ}

In univariate analysis, patients with positive TXNIP expression had a significantly longer local recurrence-free survival $(\log$ rank $(\mathrm{LR})=7.99,95 \%$ confidence interval (CI): 0.34-0.83, and $p=0.009$; Fig. 2a). When the analysis was restricted to patients who underwent breast conservative surgery, to avoid the impact of surgical management on outcome prediction, TXNIP expression retained its significant association with improved outcome ( $L R=6.67$, 95\% CI: $0.39-0.88$, and $p=0.04$; Fig. 2b). No significant association was observed between TXNIP expression and the type of local recurrence.

Positive TXNIP expression was associated with improved outcome in the group of patients treated with breast conservative surgery without postoperative radiotherapy (94/ 220) $(p=0.02)$ but not in those who were offered radiotherapy (21/93) $(p=0.5)$ (Fig. 2c, d). 

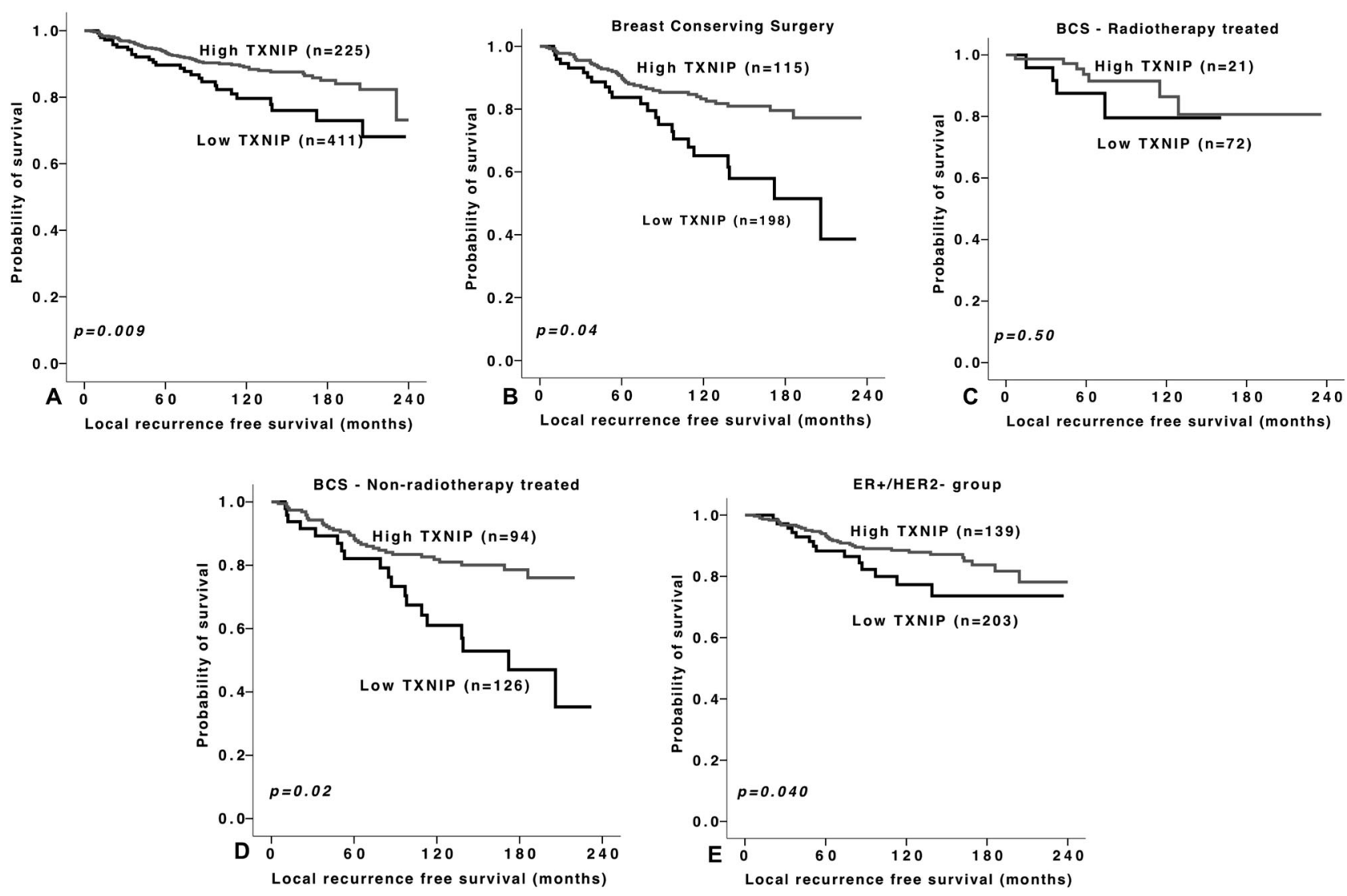

Fig. 2 Kaplan-Meier curves show that high expression of TXNIP is associated with (a) longer local recurrence-free survival in the whole series $(p=0.009), \mathbf{b}$ improved survival in patients treated with breast-conserving surgery $(p=0.04)$, $\mathbf{c}$ improved outcome in

non-radiotherapy-treated patients $(p=0.02)$, and $\mathbf{d}$ local recurrencefree survival in radiotherapy-treated patients $(p=0.50)$. e TXNIP is also associated with local recurrence-free survival when ductal carcinoma in situ stratified into luminal ER+/HER2 - $(p=0.040)$

In ductal carcinoma in situ cases treated with breast conservative surgery without postoperative radiotherapy, loss/ reduced TXNIP expression was observed in 126 cases and those showed approximately $40 \%$ recurrence rate (51/126), such patients could be candidates for radiotherapy to improve local recurrence-free survival. Low TXNIP expression was also associated with recurrence of $17 \%$ (12/72) of cases in the group of patients treated with breast conservative surgery and postoperative radiotherapy, which points out the importance of TXNIP as a predictor marker. Overall proportion of

Table 2 Multivariate Cox proportional hazards for predictors of local recurrence in pure ductal carcinoma in situ patients

\begin{tabular}{llll}
\hline Variable & HR & $95 \%$ CI & $p$ Value \\
\hline Ductal carcinoma in situ size & 0.60 & $0.46-0.89$ & $<\mathbf{0 . 0 0 1}$ \\
Ductal carcinoma in situ grade & 1.83 & $1.24-2.70$ & $<\mathbf{0 . 0 0 1}$ \\
Comedo necrosis & 0.97 & $0.54-1.73$ & 0.911 \\
TXNIP expression & 0.51 & $0.32-0.81$ & $<\mathbf{0 . 0 0 1}$ \\
\hline
\end{tabular}

$H R$ hazard ratio, $C I$ confidence interval, TXNIP thioredoxin-interacting protein

Significant $p$ values are represented in bold

recurrences after breast conservative surgery that showed low TXNIP expression were 75\% (63/84).

When molecular classes were considered, high TXNIP was associated with improved outcome in the luminal

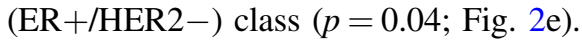

Multivariate Cox regression model, including ductal carcinoma in situ size, nuclear grade, and presence of comedo necrosis showed that high TXNIP expression was an independent predictor of good outcome in pure ductal carcinoma in situ ( $p=0.005$, hazard ratio (HR) $0.51,95 \%$ CI: 0.32-0.81) (Table 2).

\section{Discussion}

The treatment of ductal carcinoma in situ remains a challenge as the clinicopathologic features of the disease do not reliably stratify patients into distinct risk groups to guide treatment decision [23]. For this reason, some studies have attempted to risk stratify ductal carcinoma in situ based on genetic and molecular factors, including Oncotype ${ }^{\oplus}{ }^{\oplus}$ ductal carcinoma in situ score [24-28]. Although ductal 
carcinoma in situ score minimized the proportion of patients undergoing radiotherapy, the assay seems expensive and not cost-effective [29]. In fact, none of the investigated parameters has been translated to clinical practice. Therefore, there is a pressing need to identify cost-effective and robust biomarker(s) to predict outcome for ductal carcinoma in situ patients.

The role of TXNIP expression was investigated in different tissues [30-34], including locally advanced invasive breast cancer [15] and we observed that loss of TXNIP expression was associated with features of increasing aggressiveness. It is likely that the effect of TXNIP on cell growth and proliferation are cell-context-dependent and might be circumvented via activation of alternate mitogenic pathways [9], nevertheless the data are consistent with TXNIP being a tumor suppressor [30]. TXNIP expression in cancer might be downregulated through epigenetic, transcriptional, post-transcriptional, or translational mechanisms [35].

To date, there is no well-established robust publically available database for ductal carcinoma in situ mRNA or copy number aberrations. To confirm the prognostic significance of TXNIP mRNA in breast cancer and to assess the role of copy number alteration on its expression and function, we have used the METABRIC cohort. This analysis showed that loss of copy number is one of the mechanisms for downregulation of TXNIP, which is associated with aggressive behavior and poor outcome. Utilizing a large annotated series of ductal carcinoma in situ treated at a single institution, we have demonstrated that TXNIP downregulation is associated with increased aggressiveness and poor outcome in terms of shorter local recurrence-free survival. We also found downregulation of TXNIP expression in invasive disease compared to the coexisting ductal carcinoma in situ. These results support our hypothesis that TXNIP is a tumor suppressor in breast cancer.

Our results demonstrate that loss of expression of TXNIP in ductal carcinoma in situ is not only associated with parameters characteristic of poor prognosis but is also an independent predictor of recurrence. These results are in line with TXNIP functioning as a tumor suppressor that is reported to be commonly silenced by genetic or epigenetic mechanisms in cancer cells $[11,36]$.

In this study, a significant negative association between the expression of TXNIP and local recurrence in ductal carcinoma in situ was identified. A previous study indicated that TXNIP stabilizes cyclin-dependent kinase inhibitor p27, which plays a pivotal role in inhibiting cell proliferation and apoptosis resulting in $\mathrm{G}_{1}$ arrest and inhibition of cell cycle [37]. This observation may explain our finding that high TXNIP expression was associated with low-grade, low-proliferating ER-positive and HER2-negative ductal carcinoma in situ and with longer local recurrence-free survival. Independent of its interaction with TRX, TXNIP has the ability to inhibit cell cycle progression by indirectly inhibiting mammalian target of rapamycin, a regulator of cell growth and metabolism [38]. Therefore, in addition to inducing a metabolic shift important to tumor biology, downregulation of TXNIP is likely to promote cell survival, growth, invasion, and metastasis. TXNIP can also reduce tumor invasion and angiogenesis through inhibition of TRX and can directly have an impact on cell survival by promoting a pro-apoptotic environment [39, 40]. The exact mechanisms by which TXNIP exerts its tumor suppressive functions in ductal carcinoma in situ cells are not yet fully clear. Future studies of the mechanisms by which TXNIP is expressed and functions in ductal carcinoma in situ will improve our understanding of the progression to invasive disease.

In this study, a significant correlation between TXNIP expression and ER+/HER2 - status was identified. This finding is consistent with previous reports indicating that TXNIP may interrupt HER2-mediated oncogenic effect [36]. To our knowledge, few studies have reported an explicit impact of HER2 signaling on TXNIP expression. It might suggest that there is a direct link between ERBB2 and TXNIP or could be explained by the glucose theory. It is well known that cancer cells experience energetic stress and glucose deprivation especially in HER2-positive cancer cells [37]. Glucose induces TXNIP expression, and reactive oxygen species triggers the dissociation of TXNIP from TRX, leading to increased TXNIP availability [41]. Therefore, TXNIP may be induced by hyperglycemia regulated $\mathrm{TRX}$ - reactive oxygen species activity in the HER2 signaling pathway. Information regarding this mechanism will require further investigation in the future.

The current study demonstrated differential expression of TXNIP between pure ductal carcinoma in situ and ductal carcinoma in situ associated with invasive disease, which may have potential application for predicting invasive disease in ductal carcinoma in situ diagnosed on preoperative biopsy samples, after further validation. In addition, the ability of TXNIP to inhibit the major reactive oxygen species scavenger TRX, further regulate cell cycle progression and metastasis and promote apoptosis, clearly identifies TXNIP as an important therapeutic target.

In conclusion, the difficulty in implementing predictive markers for ductal carcinoma in situ remains the availability of large cohorts with consistent treatment for validation, and our study is an exception. We report that TXNIP is an independent prognostic factor and a potential tumor suppressor in breast cancer. Overexpression of TXNIP in ductal carcinoma in situ is therefore a good prognostic factor that can potentially improve ductal carcinoma in situ risk stratification for management purpose. Further functional 
studies are recommended to extensively validate the role of TXNIP as a predictor of progression to invasive disease.

Acknowledgements This research was supported and funded by the Egyptian Ministry of Higher Education and Scientific Research. We thank the Nottingham Health Science Biobank and Breast Cancer Now Tissue Bank for the provision of tissue samples. KLG supported by the Victorian Cancer Agency and a UICC Yamagiwa-Yoshida Memorial International Cancer Study Grant.

\section{Compliance with ethical standards}

Conflict of interest The authors declare that they have no conflict of interest.

\section{References}

1. Fleischer T, Frigessi A, Johnson KC, et al. Genome-wide DNA methylation profiles in progression to in situ and invasive carcinoma of the breast with impact on gene transcription and prognosis. Genome Biol. 2014;15:435.

2. Cutuli B, Bernier J, Poortmans P. Radiotherapy in DCIS, an underestimated benefit? Radiother Oncol. 2014;112:1-8.

3. Kim JY, Park K, Kang G, et al. Predictors of recurrent ductal carcinoma in situ after breast-conserving surgery. J Breast Cancer. 2016;19:185-90.

4. Schnitt SJ. The transition from ductal carcinoma in situ to invasive breast cancer: the other side of the coin. Breast Cancer Res. 2009;11:101.

5. Vincent-Salomon A, Lucchesi C, Gruel N, et al. Integrated genomic and transcriptomic analysis of ductal carcinoma in situ of the breast. Clin Cancer Res. 2008;14:1956-65.

6. Doebar SC, van den Broek EC, Koppert LB, et al. Extent of ductal carcinoma in situ according to breast cancer subtypes: a population-based cohort study. Breast Cancer Res Treat. 2016;158:179-87.

7. Carraro DM, Elias EV, Andrade VP. Ductal carcinoma in situ of the breast: morphological and molecular features implicated in progression. Biosci Rep.2014;34:e00090.

8. Gorringe KL, Hunter SM, Pang JM, et al. Copy number analysis of ductal carcinoma in situ with and without recurrence. Mod Pathol. 2015;28:1174-84.

9. Lu J, Holmgren A. Thioredoxin system in cell death progression. Antioxid Redox Signal. 2012;17:1738-47.

10. Yoshihara E, Masaki S, Matsuo Y, et al. Thioredoxin/Txnip: redoxisome, as a redox switch for the pathogenesis of diseases. Front Immunol. 2014;4:514.

11. Zhou J, Chng WJ. Roles of thioredoxin binding protein (TXNIP) in oxidative stress, apoptosis and cancer. Mitochondrion. 2013;13:163-9.

12. Kim SY, Suh HW, Chung JW, Yoon SR, Choi I. Diverse functions of VDUP1 in cell proliferation, differentiation, and diseases. Cell Mol Immunol. 2007;4:345-51.

13. Li J, Yue Z, Xiong W, et al. TXNIP overexpression suppresses proliferation and induces apoptosis in SMMC7221 cells through ROS generation and MAPK pathway activation. Oncol Rep. 2017;37:3369-76.

14. Butler LM, Zhou X, Xu WS, et al. The histone deacetylase inhibitor SAHA arrests cancer cell growth, up-regulates thioredoxin-binding protein-2, and down-regulates thioredoxin. Proc Natl Acad Sci USA. 2002;99:11700-5.

15. Woolston CM, Zhang L, Storr SJ, et al. The prognostic and predictive power of redox protein expression for anthracycline-based chemotherapy response in locally advanced breast cancer. Mod Pathol. 2012;25:1106-16.

16. Hammond ME, Hayes DF, Wolff AC, Mangu PB, Temin S. American Society of Clinical Oncology/College of American Pathologists guideline recommendations for immunohistochemical testing of estrogen and progesterone receptors in breast cancer. Arch Pathol Lab Med. 2010;6:195-7.

17. Rakha EA, Pinder SE, Bartlett JM, et al. Updated UK recommendations for HER2 assessment in breast cancer. J Clin Pathol. 2015;68:93-9.

18. Gong Y, Sweet W, Duh YJ, et al. Performance of chromogenic in situ hybridization on testing HER2 Status in breast carcinomas with chromosome 17 polysomy and equivocal $(2+)$ herceptest results: a study of two institutions using the conventional and new ASCO/CAP scoring criteria. Am J Clin Pathol. 2009; 132:228-36.

19. McCarty KS Jr, McCarty KS Sr. Histochemical approaches to steroid receptor analyses. Semin Diagn Pathol. 1984;1:297-308.

20. Curtis C, Shah SP, Chin SF, et al. The genomic and transcriptomic architecture of 2,000 breast tumours reveals novel subgroups. Nature. 2012;486:346-52.

21. Camp RL, Dolled-Filhart M, Rimm DL. X-tile: a new bioinformatics tool for biomarker assessment and outcome-based cutpoint optimization. Clin Cancer Res. 2004;10:7252-9.

22. Dolled-Filhart M, McCabe A, Giltnane J, et al. Quantitative in situ analysis of beta-catenin expression in breast cancer shows decreased expression is associated with poor outcome. Cancer Res. 2006;66:5487-94.

23. Allegra CJ, Aberle DR, Ganschow P, et al. National Institutes of Health State-of-the-Science Conference statement: Diagnosis and Management of Ductal Carcinoma In Situ September 22-24, 2009. J Natl Cancer Inst. 2010;102:161-9.

24. Solin LJ, Gray R, Baehner FL, et al. A multigene expression assay to predict local recurrence risk for ductal carcinoma in situ of the breast. J Natl Cancer Inst. 2013;105:701-10.

25. Perez AA, Balabram D, Rocha RM, da Silva Souza A, Gobbi H. Co-Expression of p16, Ki67 and COX-2 is associated with basal phenotype in high-grade ductal carcinoma in situ of the breast. J Histochem Cytochem. 2015;63:408-16.

26. Zhou W, Jirstrom K, Johansson $\mathrm{C}$, et al. Long-term survival of women with basal-like ductal carcinoma in situ of the breast: a population-based cohort study. BMC Cancer. 2010;10:653.

27. Bartova M, Ondrias F, Muy-Kheng T, et al. COX-2, p16 and Ki67 expression in DCIS, microinvasive and early invasive breast carcinoma with extensive intraductal component. Bratisl Lek Listy. 2014;115:445-51.

28. Gauthier ML, Berman HK, Miller C, et al. Abrogated response to cellular stress identifies DCIS associated with subsequent tumor events and defines basal-like breast tumors. Cancer Cell. 2007;12:479-91.

29. Raldow AC, Sher D, Chen AB, Recht A, Punglia RS. Cost effectiveness of the oncotype DX DCIS score for guiding treatment of patients with ductal carcinoma in situ. J Clin Oncol. 2016;34:3963-8.

30. Morrison JA, Pike LA, Sams SB, et al. Thioredoxin interacting protein (TXNIP) is a novel tumor suppressor in thyroid cancer. Mol Cancer. 2014;13:62.

31. Nishizawa K, Nishiyama $H$, Matsui $Y$, et al. Thioredoxininteracting protein suppresses bladder carcinogenesis. Carcinogenesis. 2011;32:1459-66.

32. Tome ME, Johnson DB, Rimsza LM, et al. A redox signature score identifies diffuse large B-cell lymphoma patients with a poor prognosis. Blood. 2005;106:3594-601.

33. Sheth SS, Bodnar JS, Ghazalpour A, et al. Hepatocellular carcinoma in Txnip-deficient mice. Oncogene. 2006;25:3528-36. 
34. Goldberg SF, Miele ME, Hatta N, et al. Melanoma metastasis suppression by chromosome 6: evidence for a pathway regulated by CRSP3 and TXNIP. Cancer Res. 2003;63:432-40.

35. Zhou J, Yu Q, Chng WJ. TXNIP (VDUP-1, TBP-2): a major redox regulator commonly suppressed in cancer by epigenetic mechanisms. Int J Biochem Cell Biol. 2011;43:1668-73.

36. Nie W, Huang W, Zhang W, et al. TXNIP interaction with the Her-1/2 pathway contributes to overall survival in breast cancer. Oncotarget. 2015;6:3003-12.

37. Elgort MG, O'Shea JM, Jiang Y, Ayer DE. Transcriptional and translational downregulation of thioredoxin interacting protein is required for metabolic reprogramming during G(1). Genes Cancer. 2010;1:893-907.
38. Jin HO, Seo SK, Kim YS, et al. TXNIP potentiates Redd1induced mTOR suppression through stabilization of Redd1. Oncogene. 2011;30:3792-801.

39. Dunn LL, Buckle AM, Cooke JP, Ng MK. The emerging role of the thioredoxin system in angiogenesis. Arterioscler Thromb Vasc Biol. 2010;30:2089-98.

40. Welsh SJ, Bellamy WT, Briehl MM, Powis G. The redox protein thioredoxin-1 (Trx-1) increases hypoxia-inducible factor 1alpha protein expression: Trx-1 overexpression results in increased vascular endothelial growth factor production and enhanced tumor angiogenesis. Cancer Res. 2002;62:5089-95.

41. Schroder K, Zhou R, Tschopp J. The NLRP3 inflammasome: a sensor for metabolic danger? Science. 2010;327:296-300. 